\title{
Jennifer Eickelmann
}

\section{МHATE SPEECH \\ UND VERLETZBARKETT \\ IM DIGITALEN ZEITALTER}

Phänomene mediatisierter Missachtung aus Perspektive der Gender Media Studies

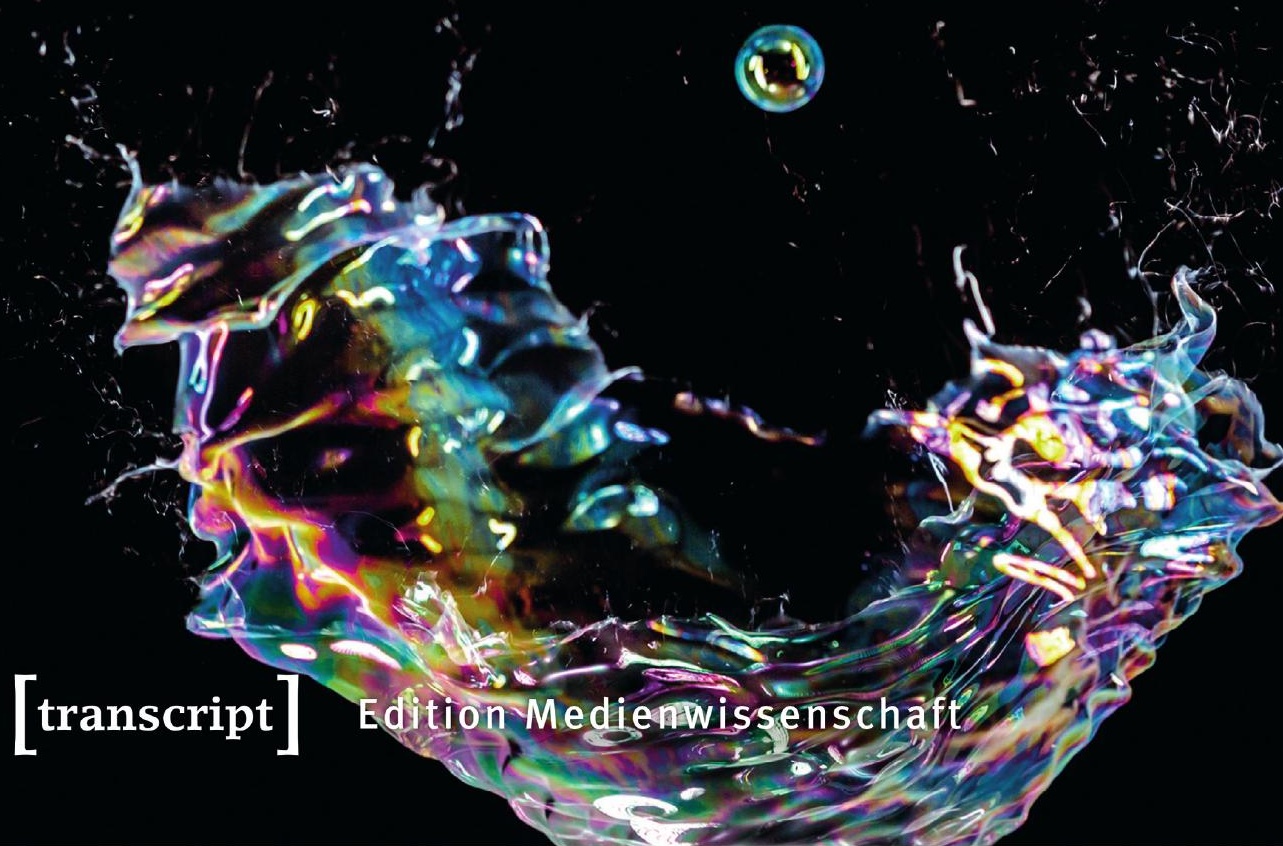




\section{Aus:}

Jennifer Eickelmann

\section{»Hate Speech« und Verletzbarkeit im digitalen Zeitalter Phänomene mediatisierter Missachtung aus Perspektive der Gender Media Studies}

August 20I7, 332 Seiten, kart., farb. Abb., 32,99 €, ISBN 978-3-8376-4053-3

Die Debatten um Hate Speech im Internet zeugen von der Brisanz der Frage, welche Verletzungsmacht diffamierenden Adressierungen inhärent ist: Handelt es sich um einen rein zeichenhaften Ausdruck freier Rede oder um einen >realen< Gewaltakt?

Aus einer dualismuskritischen Perspektive entwickelt Jennifer Eickelmann ein Konzept mediatisierter Missachtung, das sich diesem Entweder-oder verweigert. Entlang materialreicher Analysen zeigt sie die Kontingenz dieser Kommunikationen im Spannungsfeld von Realität/Virtualität auf und legt dar, welche Bedeutung der Kategorie Gender und dem Medialen bei der Konstitution und Wirkmacht mediatisierter Missachtung zukommt.

Jennifer Eickelmann (Dr. phil.) ist als wissenschaftliche Mitarbeiterin am Institut für Soziologie der TU Dortmund tätig. Die kulturwissenschaftlich ausgerichtete Medienwissenschaftlerin und Soziologin promovierte am Institut für Medienwissenschaft der Ruhr-Universität Bochum. Ihre Forschungsschwerpunkte sind Gender Media Studies, Theorie und Ästhetik des Digitalen, Performativitätstheorie und die Analyse kultureller Praxis, diskurstheoretische Subjektivationstheorie sowie Museumsforschung.

Weitere Informationen und Bestellung unter: www.transcript-verlag.de/978-3-8376-4053-3

(C) 2017 transcript Verlag, Bielefeld 


\section{Inhalt}

\section{Danksagung | 9}

$1 \quad$ Einleitung | 13

2 Perspektivierung \& Vorgehensweise 29

2.1 >Neue $<$ Materialisierungen? Zur destruktiven Kraft hybrider Praxis im Netz | 29

2.1.1 Problemaufriss \& Programmatik | 29

2.1.2 Eine Kritik des Agentiellen Realismus nach Karen Barad | 32

2.1.3 Eine Kritik der Symmetrischen Anthropologie nach Bruno Latour, mit einem Ausflug zu den Existenzweisen I 41

2.1.4 Positionierung: Judith Butler im Lichte des New Materialism I 51

2.2 Virtualität im Spiegel von Realität und Fiktion I 61

2.3 Zusammenfassung I 69

2.4 Zuspitzung: Diffraktion als Methode 171

\section{Subjektivation im Netz I 75}

3.1 Eine Geschichte des Internets I 75

3.2 Subjektivation in der Kultur der Dauerkonnektivität I 87

3.2.1 Digitale Hypermedialität als Modus des Performativen I 90

3.2.2 Daueradressierbarkeit, Beschleunigung, Distanzabbau I 96

3.2.3 Mensch oder Maschine? Social Bots als intraaktives Phänomen I 103

3.2.4 Unterwerfung und Subjektwerdung im Netz I 111

3.2.5 Übertragung: Mediatisierte Empörungswellen als Subjektivationsmodus I 114

\section{Hassrede vs. Redefreiheit - Ein Irrweg I 117}

4.1 Hassrede - Redefreiheit: Universalpragmatik und Juridifizierung I 117

4.1.1 Zum Dualismus I 118

4.1.2 Konsensualer Universalismus vs. Digitale Hypermedialität I 124

4.1.3 (Ent-)Politisierung des Internets I 127

4.1.4 Das Netz als entgrenztes Medium und die Frage nach dem Recht I 136

4.1.5 Von >Tätern〈 und 〉Opfern〈 I 138

4.2 Jenseits der dualistischen Falle: Postsouveräne Subjektivität im Netz | 139

4.3 Zusammenfassung | 146 
5 Diskursive Störfigurationen: Von Trollen, Cybermobbing \& Shitstorms | 149

5.1 Einführung | 149

5.2 Die Figur(ation) des Troll(en)s | 151

5.1.1 Der Troll als männliche Figur | 153

5.1.2 Trolling und Hacking | 156

5.1.3 Kontingenz: Trollen als ernstes Spiel I 158

5.2 Cybermobbing und Cyberbullying | 163

5.2.1 Cybermobbing als Gewaltakt, Internet als Waffe I 163

5.2.2 Materielle (Re-)Konfigurationen der Welt durch Forschungsdesigns | 166

5.2.3 Täterinnen in der Virtualität | 168

5.3 Shitstorms | 172

5.3.1 Der Shitstorm als Pseudo-Anglizismus | 172

5.3.2 Öffentlichkeit und Aufmerksamkeitsökonomie | 176

5.4 Zusammenfassung | 183

$6 \quad$ Materialisierungen | 187

6.1 Diffraktive Fallanalysen | 187

6.2 Gefährdetes Leben in der Spielkultur: Der Fall Anita Sarkeesian I 190

6.2.1 Bedingungskonstellationen: \#Gamergate als Interferenzmuster | 190

6.2.2 Multimodale Missachtungen I 211

6.2.3 Rekonfigurationen: Von neuen Kontexten, Zeugen und Kompliz_innen I 221

6.2.4 Zusammenfassung | 227

6.3 Über den Tod hinaus belebt: Der Fall Amanda Todd I 228

6.3.1 Fokus: Note Card Stories auf YouTube im Modus des Sentimentalen I 228

6.3.2 Subjektivation post mortem I 246

6.3.3 Rekonfigurationen: Zur Sichtbarmachung von Verletzbarkeit I 268

6.3.4 Zusammenfassung | 274

6.4 Resümee: Mediatisierte Missachtung und Verletzbarkeit I 275

$7 \quad$ Fazit und Ausblick | 279

Literatur und weitere Quellen | 287

Abbildungen | 327 


\section{Einleitung}

Und so kann man nachdenklich und folglich mit einem gewissen Sinn für Bescheidenheit sagen, dass ich zu Beginn mein Verhältnis zu dir bin, zweideutig angeredet und anredend, einem $>$ Du ausgeliefert, ohne das ich nicht sein kann und von dem mein Überleben abhängt.

BUTLER 2014A: 110, HERV. I. ORIG.

Auf der Technologie-Messe CEATEC ${ }^{1}$ in Japan schaut eine junge Frau im Oktober 2015 ungläubig und zögerlich, zugleich jedoch fasziniert und neugierig in einen Spiegel. Ihr offener Blick hat eine klare Richtung, die zugleich das für sie sichtbar gewordene Bild erzeugt: sie selbst. Doch was macht diese Situation des Blickens in den Spiegel so bemerkenswert? Die Antwort ist simpel, aber erstaunlich: Das \Selbst im Spiegel reflektiert nicht das \Selbst vor dem Spiegel. Der japanische Elektronikkonzern PANASONIC hat auf der CEATEC seinen >Interactive Mirror^vorgestellt, der im Jahr 2017 im Handel erhältlich sein soll (gfu Consumer \& Home Electronics GmbH 2015). Die Farbe des Lippenstiftes, die Nuance des Make-ups sowie des Lidschattens - aber auch der Schnitt und die Länge des Bartes werden mach- und modifizierbar. Die reflexive Selbstbezüglichkeit des Spiegelbildes, d.h. seine Eigenschaft, das Selbst vor dem Spiegel im Spiegelbild zu reflektieren, wird damit technologisch aufgebrochen, was eine technologisch bedingte Verschiebung des Selbst zur Folge hat. Eben jene technologische Aufbrechung verändert das klassische Verständnis vom Spiegels und seiner Funktion als Ermöglichung menschlicher Selbstbezüglichkeit wie menschlichen Selbstbewusstseins.

1 Abkürzung für >Combined Exhibition of Advanced Technologies`. 
Dem klassischen Spiegel wird, Lacan folgend, der Effekt zugeschrieben, Bildner der Ichfunktion zu sein (Lacan 1973). Ihm geht es um die zentrale Frage, wie der Mensch sich seiner selbst bewusst wird, so dass er ein >Ich` entwickeln kann. Auf der Grundlage des im Spiegel erblickten Selbstbildes entwickelt sich Lacan zufolge das Imaginäre, d.h. das Bildhafte im Psychischen (ebd.: 63). Das Subjekt wandelt sich in Bezug auf sein Verhältnis zu sich selbst also durch die Aufnahme dieses Bildhaften (ebd.: 64). Jenes Bildhafte im Psychischen konstituiert sich demnach also erst mittels des Spiegelbildes, das eine Reflexion des Abgebildeten darstelle (ebd.: 63). Dem Spiegel liegt klassischerweise folglich die Metapher der Reflexion zugrunde, die davon ausgeht, dass der Spiegel die Realität einfängt und zurückwirft. Das Werden des Subjekts als ein >Ich〈bzw. als ein >Selbst< beruht demnach auf der Vorstellung, sich selbst vollständig zu sehen, d.h. sich selbst als eine Einheit zu erfahren (ebd.: 64f.). Daraus folgt, dass das Erkennen des Selbst im Spiegel auf einer »triumphalen Übung « (Lacan, zit. n. Bitsch 2009: 69) beruht, bei der es um das Ergreifen einer ungezügelten Herrschaft des Ichs geht (Bitsch 2009: 70). Dennoch gilt: Das imaginäre Selbst im Spiegel ist grundlegend auf den menschlichen Blick bzw. die Beobachtung angewiesen: Das Spiegelbild bezieht sich damit auch unweigerlich auf die Beobachtungsbedingungen selbst, die mit dem Spiegel beobachtbar werden (Esposito 2000: 287).

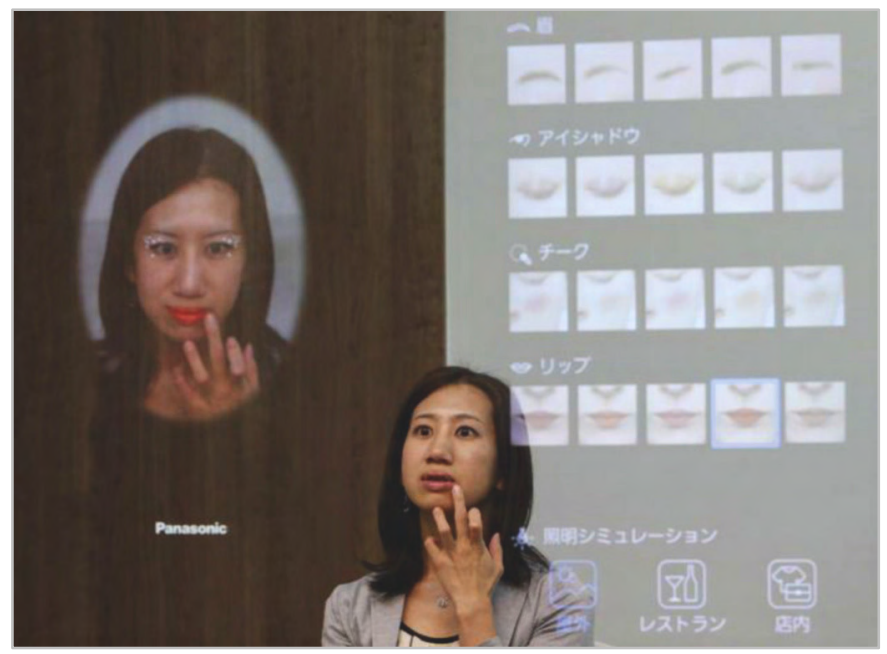

Abbildung 1: Interaktiver Spiegel der Firma Panasonic, Makuhari, Japan

Der interaktive Spiegel stellt nun aufgrund seiner Technizität das Moment menschlicher Herrschaft in Frage - der Schein der Souveränität gerät ins Wanken. Gleichzeitig und damit untrennbar verbunden stellt der interaktive Spiegel 
aufgrund seiner Modifikationsoptionen, welche die Nutzer_innen selbst auswählen und anwenden können, ein Phantasma souveräner Subjektivität performativ her, welches die vermeintliche Verfügungsgewalt über sich selbst technologisch ausweitet. Entsprechend drängen sich zwei Fragen auf: erstens die Frage nach der Selbstbezüglichkeit und zweitens die Frage nach der Rolle der Beobachtungsbedingungen.

Die Frage nach der Selbstbezüglichkeit aufgreifend scheint es wichtig, zuerst kurz die Apparatur des >Interactive Mirror` zu beschreiben: Bei der Apparatur handelt es sich zugleich um einen Spiegel, eine Projektionsfläche und in Teilen um eine Kamera. Der interaktive Spiegel reflektiert somit nicht etwa das Selbst vor dem Spiegel, sondern er zeichnet ein Bild, welches ein Morphing aus dem Spiegelbild und der Kameraaufzeichnung darstellt. In der Mitte des Spiegels befindet sich das ovale »augmented-reality-portal« (Wong 2015). ${ }^{2}$ In diesem Bereich ist das Gesicht zu platzieren, denn dort befindet sich die integrierte Kamera. Nun kann das Experimentieren mit dem (vergeschlechtlichten) Selbst irgendwo zwischen der Realität und der Virtualität mittels eines Knopfdrucks gestartet werden (ebd.). Die dem interaktiven Spiegel zugrundeliegende Software ermöglicht es, das Spiegelbild und das von der Software erzeugte virtuelle Bild übereinanderzulegen. Damit gehen das Spiegelbild und das virtuelle Bild ineinander auf. Der Blick der Kamera und der Blick der Betrachterin verschmelzen miteinander. Diese Verschiebungen zugrunde legend, gerät die Metapher der Reflexion zur Beschreibung der hier vorliegenden Szenerie an ihre Grenzen, da die Technizität des Spiegels dazu führt, dass nicht etwa die Realität vor dem Spiegel zurückgeworfen, d.h. reflektiert wird, sondern es wird ein intraaktives Bild, bestehend aus Spiegel- und Kamerabild, geschaffen. In den Worten Donna Haraways ist $»[\ldots]$ reflexivity [...] not enough to produce self-visibility« (Haraway 1997: 268).

Wer ist es also, den oder die ich im Spiegel sehe? Das Subjekt im Spiegel steht zwar in einer Relation zum >realen` Subjekt, und dennoch ist es nicht mit ihm identisch. Es ergibt sich vielmehr ein virtuelles Subjekt, welches sich im Spannungsfeld unterschiedlicher Realitätsdimensionen irgendwo zwischen der Realität und der Fiktion ansiedelt. Der interaktive Spiegel erzeugt in Abhängigkeit des >realen` Subjekts ein >virtuelles`Subjekt und verwirrt damit die vermeintlich eindeutige Trennung zwischen Realität und Virtualität. Nun hatte es vor der Präsentation von PANASONIC bereits andere Spiegel gegeben, die das

2 Der Begriff >Augmented Reality< meint eine interaktive Technologie, die virtuelle Elemente und die physische Umgebung überlagert (vgl. weiterführend Alkhamisi/Monowar 2013). 
Spiegelbild modifizierbar machen. Ebenso gibt es bereits seit Längerem Apps, mit denen das eigene Kamerabild modifiziert werden kann. ${ }^{3}$ Das Innovative des >Interactive Mirrors besteht jedoch insbesondere darin, dass er nicht lediglich einen bestimmten Bereich, d.h. den sichtbaren Bereich des Spiegels bzw. der Kamera erfassen und modifizieren kann. Die Sichtbarmachung geht weit darüber hinaus: Mithilfe von Sensoren im Bett und auf dem Stuhl vor dem Spiegel errechnet die Apparatur das Gewicht, den Body-Mass-Index sowie die für den Tag empfohlene Kalorienmenge (gfu Consumer \& Home Electronics GmbH 2015). Der Spiegel und seine Software sind Teil der Serie »A Better Life, A Better World «, in der PANASONIC zahlreiche Produkte präsentiert, die in die Wohnumgebung integriert werden, wie vernetzte Haushaltsgeräte und intelligente Klima-, Klang- und Beleuchtungssysteme, abgestimmt auf den menschlichen Biorhythmus (Businesswire 2015). Das, was im Spiegel sichtbar wird, ist somit immer auch ein durch gespeicherte und für das Unternehmen wertvolle Daten vermessenes, kontrolliertes, diszipliniertes wie ökonomisiertes Subjekt. Die aufgezeichneten Daten münden in Kosmetik- und Ernährungshinweisen, die mithilfe der Spiegel-Oberfläche online in eine Warentransaktion transformiert werden können (vgl. gfu Consumer \& Home Electronics; vgl. Antona/Stephanidis 2015: 357ff.). Außerdem kommt dem Bild im Spiegel eine unmittelbare Bedeutung für die Realität `vor` dem Spiegel zu, da die Funktionalität der Apparatur überhaupt nur darin besteht, dass das Ausprobieren in der Virtualität, z.B. mit der Frage, welcher Lidschatten >zu mir passt‘, Konsequenzen für die reale Lebensführung, darunter die Wahl des >richtigen< Lidschattens, haben soll. Die Selbstbezüglichkeit des interaktiven Spiegels stellt sich also bei genauerer Betrachtung als eine technologisch und ökonomisch bedingte Selbstbezüglichkeit heraus. Davon ausgehend kommen wir zur zweiten aufgeworfenen Frage nach den Beobachtungsbedingungen des Blicks in den Spiegel.

Elena Esposito zufolge repräsentiert der klassische Spiegel die Realität aus einem anderen Blickwinkel und thematisiert somit unmittelbar die Beobachtungsbedingungen selbst (Esposito 2000: 287). Damit ist gemeint, dass der Spiegel die Beobachtungssituation sichtbar macht. Während allerdings beim klassischen Spiegel die Beobachtungsbedingung besonders der gerichtete Blick innerhalb einer spezifischen Beobachtungssituation ist, stellt sich die Lage in Bezug auf den interaktiven Spiegel weitaus komplexer dar. Der Blick in den interaktiven Spiegel, d.h. in einen Spiegel, eine Kamera und auf eine Projektionsfläche, eröffnet zusätzlich einen Blick auf die Mitgestaltungsmöglichkeiten des Spiegelbildes. Während die kosmetischen Operationen am Spiegel bzw. Bildschirm

3 Wie beispielsweise die App Snapchat, die seit 2011 auf dem Markt ist. 
selbst erst vorgenommen werden müssen, wird Aktivität unmittelbar zur Bedingung des virtuellen Bildes (vgl. Esposito 2000: 287). Die Lippenstiftfarbe, das Make-up oder auch die Bartfrisur müssen mithilfe einer Fernbedienung ausgewählt werden (vgl. Panasonic USA 2015). Die Bezugsrealität des Realen und die Bezugsrealität des Virtuellen fließen ineinander über und verwirren sich gegenseitig. Damit sind jene Handlungen der Subjekte vor dem Spiegel unweigerlich medientechnologisch bedingt: Nur in Abhängigkeit der Angebote der Software können bestimmte Kosmetika und Erscheinungsbilder ausgewählt werden. Das virtuelle Selbst beruht somit eben nicht nur auf dem gerichteten Blick des Subjekts, sondern auch auf dem gerichteten Blick der Kamera sowie jener Sichtbarmachung spezifischer Produkte mithilfe der Software. Mit den entstehenden Daten und der Software sind nun Modifikationen möglich, die das virtuelle Subjekt erst konstituieren.

Dieses einführende Beispiel, über das sicherlich noch viel mehr zu sagen wäre, soll an dieser Stelle dem Zweck dienlich sein, auf die der vorliegenden Arbeit zugrunde liegende Annahme zum Verhältnis von Medientechnologien und Subjektivität zu verweisen. Wie am Beispiel des Interactive Mirrors deutlich wurde, handelt es sich bei der Virtualität um eine Realitätsdimension, die nicht unabhängig von der Realität funktioniert, sondern auch auf Subjekte der Realität angewiesen ist. Insofern kann die Subjektkonstitution im Kontext von Medientechnologien kein Prozess sein, der innerhalb einer virtuellen Blase stattfindet. Vielmehr geht es in der vorliegenden Arbeit um die Bezüglichkeit unterschiedlicher Realitätsdimensionen, ihrer Wechselwirkungen, Mischverhältnisse, Irritationen, oder anders: Interferenzen (Bath u.a. 2013). In Anlehnung daran wird in der vorliegenden Arbeit die These aufgestellt, dass internetbasierte Dienste und Diskurse einen noch zu diskutierenden Effekt auf die Materialisierung des Lebens insgesamt haben.

Jene These kommt nicht umhin, historische Entwicklungen zu diskutieren: Die Metapher des Spiegels diente insbesondere in den 1990er Jahren dazu, die Virtualität als »Spiegel für die Kommunikation« (Esposito 2000: 289) zu beschreiben. Allerdings ist mittlerweile deutlich geworden, dass es sich bei dem Virtuellen eben nicht um eine Reflexion der Realität handeln kann, sondern die medientechnologische Bedingtheit des Virtuellen zur Folge hat, dass sie die Realität modifiziert, verschiebt und neue Perspektiven erlaubt, d.h. die Komplexität der Beobachtung steigert (ebd.: 289). Donna Haraway hat eine andere Metapher zur Beschreibung dieser Verschiebungen und Anreicherungen vorschlagen, die im Laufe der Arbeit eine zentrale Rolle spielen wird: Diffraktion (Haraway 1997: 268). Der Begriff bzw. die Methodologie der Diffraktion, ebenfalls eine Metapher aus der Optik, beschreibt den Prozess der Beugung von Wellen, 
wodurch sich spezifische Beugungsmuster ergeben, die auch häufig mit dem Begriff Interferenz(muster) beschrieben werden (Bath u.a. 2013: 7ff.). ${ }^{4} \mathrm{Um}$ wieder zum eingangs erläuterten Beispiel zu kommen: So betrachtet stellt das Bild in dem interaktiven Spiegel ein Interferenzmuster dar, welches das Ergebnis des Interferierens von Realität und Virtualität sowie von Subjektivität und Technizität darstellt. Die Metapher der Diffraktion ist entsprechend gegen die Vorstellung reiner`Selbstbezüglichkeit verortet, wie es die Metapher der Reflexion suggeriert, und hat damit eine Perspektivverschiebung zum Ziel. Es geht nicht mehr um Abbilder, sondern um Interferenzbilder, welche die Trennung von Original und Abbild, Realität und Virtualität ad absurdum führen.

Die Serie »A Better Life, A Better World « von PANASONIC kann als Sinnbild für die fortschreitende Technisierung bzw. Digitalisierung des Lebens insgesamt betrachtet werden. Längst wurde die Angst vor Überwachung durch moderne Informationstechnologien von einer umfassenden, produktiv-machtvollen Integration eben dieser Technologien in die Lebensführung von Subjekten abgelöst (vgl. Foucault 2005a: 890; Barad 2012a, b: 32ff.; Kember/Zylinska 2012: xiii, 129ff.). Damit einher geht das Faktum, dass unterschiedlichste Plattformen, Kommunikationskanäle, Datenspeicherungs- und -auswertungstools längst die performative Herstellung des eigenen Selbst bedingen (vgl. Becker/Eickelmann 2009; Bublitz 2010; Reichert 2015). Umgekehrt wird durch die performativ wirksame Nutzungspraxis in einem fortlaufenden Prozess aber auch erst das hergestellt, was als Medium erscheint (vgl. Seier 2007: 69ff.; vgl. Völker 2010: 325). Dauerkonnektivität und die ihr zugrunde liegenden technologischen und ökonomischen Infrastrukturen von Internettechnologien (van Dijck 2012: 7) werfen damit nicht zuletzt die Frage auf, wo der Mensch endet, oder anders ausgedrückt, inwiefern die vermeintliche Grenze zwischen dem, was als das Menschliche gilt, und jenem, was als das Technologische bzw. Ökonomische gilt, überhaupt aufrechtzuerhalten ist. Medientechnologien - verstanden als technische Apparaturen, symbolische Verfahren sowie Praktiken der Form(ier)ung, mit denen sie operieren (Bublitz 2010: 8, Fn 2) - verlängern sich gewissermaßen in die Selbstverhältnisse der Subjekte, ihre sozialen Beziehungen wie auch in ihre Körper hinein (ebd.). Die Allgegenwärtigkeit internetbasierter Dienste führt vor Augen, dass Medientechnologien sich nicht als von ihren Nutzer_innen getrennte Entitäten beschreiben lassen, sondern diese Technologien das Leben derart durchziehen, dass Subjektivierungsprozesse immer auch im Kontext ihrer techno-medialen Bedingtheit diskutiert werden müssen:

4 Vgl. vertiefend Kap. 2.1.2. 
»Die Digitalisierung und Synthetisierung stellt $[\ldots]$ nach der Kopernikanischen Wende, der Evolutionstheorie von Darwin und der Psychoanalyse Freuds die vierte narzisstische Kränkung des Menschen bzw. Subvertierung des anthropozentristischen Humanismus dar« (Deuber-Mankowsky 2007a: 278).

Vor dem Hintergrund der durch Digitalisierung und Synthetisierung fragil gewordenen Grenze zwischen dem Menschlichen und dem Technologischen etablieren sich mediatisierte Praktiken der Subjektivation, die darauf angelegt sind, sich der eigenen Existenz zu vergewissern (vgl. Pauleit 2001; Bublitz 2010: 11). ${ }^{5}$ Die Frage der Genese des Subjekts ist spätestens seit der Veröffentlichung von Judith Butlers Gender Trouble. Feminism AND THE Subversion OF IDENTITY (1990) untrennbar mit "grundlegenden Kategorien des Geschlechts, der Geschlechtsidentität und des Begehrens als Effekte einer spezifischen Machtformation« (Butler 2012a: 9) verbunden. Wenn sich die »)Geschlechtsidentität nicht aus den politischen und kulturellen [und ergänzend: medientechnologischen, Erg. d. Verf.] Vernetzungen herauslösen lässt, in denen sie ständig hervorgebracht und aufrechterhalten wird « (Butler 2012a: 18), dann gilt es, danach zu fragen, wie sich die wechselseitige Hervorbringung von Subjektivität im Spannungsfeld von Gender und Medientechnologien in der Kultur der Dauerkonnektivität gestaltet (Seier 2009; van Dijck 2012). Davon ausgehend, dass die Evidenz eines vermeintlich natürlichen Körpers ein Effekt »eines durch Normen regulierten Settings« (Peters 2002) darstellt, gerät die medientechnologische Eingebundenheit vergeschlechtlichter Körper als medientechnologische Bedingtheit von Subjektivationsprozessen in den Blick. Im Zuge von Dauerkonnektivität und Dauerpräsenz im Netz werden vergeschlechtlichte Subjektivitäten ausgehandelt, die sich untrennbar in und mit der medialen Öffentlichkeit konstituieren (vgl. Bublitz 2010: 8). So wundert es kaum, dass im Netz auffällig viele Bilder des Interactive Mirrors zu finden sind, die Frauen mit einem Bart ebenso wie Männer mit farbenfrohen Kosmetika als machbares `Kuriosum` präsentieren (Wong 2015). Durch zeitliche und räumliche - und damit unmittelbar zusammenhängend: körperliche - Entgrenzung verändern medientechnologische Prozesse nicht nur die Wahrnehmung, sondern auch die Existenz insgesamt. Jenseits physischer Anwesenheit können jederzeit und allerorts Adressierungen stattfinden, was die Veränderung der »klassischen Zeit der Massenmedien« (Angerer 2014) zur Folge hat. Marie-Luise Angerer hat darauf hingewiesen, dass Gilles Deleuze die Veränderung von der Disziplinar- zur Kontrollgesellschaft ebenfalls

5 Die Begriffe >Subjektivation` und `Subjektivierung` werden im Laufe der Arbeit synonym verwendet. 
im Kontext der Veränderung von Temporalität betrachtet hat (ebd.: 351). Deleuze folgend, lassen sich zudem - und damit untrennbar verbunden - räumliche Entgrenzungen sowie eine substanzielle Entgrenzung des eigenen Seins konstatieren. Daraus folgert Deleuze, dass »der Mensch der Kontrolle eher wellenhaft ist, in einem kontinuierlichen Strahl, in einer Umlaufbahn« (Deleuze 1993: 259).

Über die Subjektkonstitution im Kontext digitaler Medientechnologien ist bislang mit unterschiedlichen Schwerpunktsetzungen geschrieben worden. Insbesondere die Frage nach der Etablierung >neuer Kulturpraktiken der Selbstkonstitution und Selbstinszenierung im Kontext medientechnischer Verfahrensweisen kann als Fokus dieser Debatte bezeichnet werden (Reichert 2008; Bublitz 2010; Pietraß/Funiok 2010; Carstensen/Schachtner/Schelhowe/Beer 2014; Hitzler 2014; Paulitz/Carstensen 2014). Damit ist das Internet auch eine Arena, in der die Grenzen der Sicht- und Anerkennbarkeit in unterschiedlichen Realitätsdimensionen ausgehandelt werden. Obwohl Vergeschlechtlichung im Interactive Mirror entsprechend als künstliches, d.h. zu erzeugendes Prinzip sichtbar wird, folgt jedoch aus diesem Umstand keineswegs unmittelbar eine Abkehr von begrenzenden, naturalisierenden Vorstellungen von Geschlecht jenes Wahrheitsregimes der Realität, welches Subjekten einen ontologischen Status zuweist (Butler 2014a: 34). Das Verhältnis von Virtualität und Realität im Kontext von Gender und damit zusammenhängenden Möglichkeitsräumen und Anerkennungsstrukturen sowie deren Beschneidungen und Limitationen stellt sich also weitaus komplexer dar als bisher angenommen.

\section{Zum gegenständlichen Fokus der Arbeit und Klärung von Begrifflichkeiten}

Davon ausgehend, dass Subjekte medientechnologisch bedingt sind, lassen sich spezifische Internetphänomene folglich auch darauf hin untersuchen, inwiefern sie Subjekte ihrer Legitimation berauben, sie einschränken und sogar vernichten können.

Jene Heilsversprechen, welche die Geschichte des Internets seit den späten 1980er Jahren bzw. 1990er Jahren insbesondere im Kontext cyberfeministischer Bestrebungen prägten und das Internet als utopischen Raum imaginierten, der jenseits des realen - heißt: snatürlichen « - Körpers funktionieren und damit bislang unvorstellbare Möglichkeitsräume für Multirollenexistenzen bereitstellen sollte (VNS Matrix 1996; Turkle 1999), haben sich nicht eingelöst (Eickelmann 2012). Entgegen der Vorstellung, dass sich im Internet Existenzen etablieren, die jenseits >realer`Verworfenheiten existieren können (Turkle 1984: 10), tritt nun deutlich zutage, dass es sich bei der Virtualität des Internets eben nicht um eine Sphäre handelt, die jenseits >realer<, historisch gewachsener Zusammenhänge 
funktioniert. Das Internet ist kein anderer Raum, stattdessen werden Prozesse der Grenzüberschreitung mittels mediatisierter Missachtung, d.h. konkreter Diffamierungen, Beleidigungen, bis hin zu Vergewaltigungs- und Morddrohungen versucht, gewaltvoll zu sanktionieren. Dieses spezifische Phänomen zusammenfassend lässt sich mediatisierte Missachtung als potenziell gewaltförmige Praxis multimodaler Herabsetzung genauer begreifen. Das hier zu diskutierende Phänomen wird in der öffentlichen Auseinandersetzung zumeist mit dem Begriff >Shitstorm « als Ausdrucksform der >digitalen Wutbürger_innen (vgl. Lobo 2013; Herbold 2014). Jenseits des `Shitstorms` als Figur mediatisierter Missachtung tauchen in unterschiedlichen Diskursen mit jeweils spezifischen Lagerungen allerdings noch weitere Figurationen auf, wie >Cybermobbing` und >Trollen . Damit wird deutlich, dass mediatisierte Missachtung als Sammelbegriff spezifische figurale Konstellationen subsumiert, die zumeist gemeinsame Adressat_innen aufweisen und sich so als kaum einzugrenzendes Bündel missachtender Adressierungen artikulieren. Um die diskursiven Einschreibungen und Engführungen der jeweiligen Figurationen nicht zu reproduzieren, sondern diese auf einer Metaebene diskutieren zu können, schreibe ich im Folgenden von $>$ mediatisierten Empörungswellen $\measuredangle$. Mediatisierte Missachtung wird entsprechend als ein konstitutives Moment mediatisierter Empörung gefasst. Dieser Meta-Begriff erlaubt auf der einen Seite die Medienspezifizität des Phänomens zu betonen, denn eine systematische Perspektivierung und Interpretation der Medialität des Phänomens fehlt im wissenschaftlichen Diskurs bislang (vgl. Pörksen/Detel 2012; Han 2013). Zudem erlaubt der Begriff mediatisierte Empörung, ohne Begrifflichkeiten auszukommen, denen von Beginn an normative Wertungen eingeschrieben sind. Der Begriff mediatisierte Missachtung als Teil mediatisierter Empörung abstrahiert in diesem Sinne von unterschiedlichen

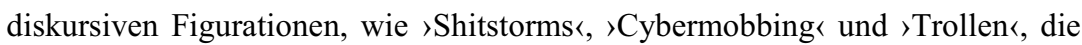
nicht vorausgesetzt werden, sondern zum Gegenstand der Untersuchung gemacht werden. Zudem verweist der Begriff >mediatisierte Empörung`zugleich auf die affektive Ebene eben jenes Phänomens, ohne eine normative Wertung des zu Beschreibenden mitzuführen. Damit soll zugleich darauf verwiesen werden, dass mediatisierte Empörungswellen zwar mediatisierte Missachtung als konstitutives Element in sich tragen, allerdings nicht gänzlich in ihr aufgehen, denn spezifische Formen der Solidarisierung mit Adressierten sind ein ebenso wichtiges Element mediatisierter Empörung. Der Schwerpunkt der vorliegenden Arbeit liegt allerdings insbesondere bei mediatisierter Missachtung, wenn auch auf die Frage nach Solidarisierungsprozessen innerhalb mediatisierter Empörungswellen nicht verzichtet werden kann und soll. Doch wie lässt sich mediatisierte Missachtung genau fassen? 
Mediatisierte Missachtung wird hier verstanden als Miss-Achtung, d.h. als medientechnologisch bedingte Zurückweisung und Herabsetzung, die Ausschlüsse produziert und damit den Möglichkeitsraum für (Über-)Lebensfähigkeit begrenzt. Mediatisierte Missachtung stellt somit eine Bedrohung für Subjekte im existenziellen Spannungsfeld von Realität und Virtualität dar, deren Effektivität nicht im Vorhinein abgeschätzt werden kann. Das Funktionieren der Bedrohung, d.h. ihre performative Effektivität, ist dabei unmittelbar an einen durch Teilöffentlichkeiten im Netz hergestellten Schauplatz der Macht geknüpft (Butler 2006: 25), welcher im Kontext digitaler Hypermedialität von technologischen wie ökonomischen Infrastrukturen ebenso mitbestimmt wird, wie von historisch gewachsenen und performativ hergestellten diskursiven Formationen.

Mediatisierte Missachtung artikuliert sich mittels Diffamierungen, Herabsetzungen, Beleidigungen - mit den Worten Judith Butlers: Excitable Speech (Butler 1997) - und ist eine konstitutive Ausdrucksform mediatisierter Empörungswellen (Han 2013; Pörksen/Detel 2012), die im Fokus der vorliegenden Arbeit stehen soll. Als performativer Sprechakt ist die mediatisierte Empörungswelle in die >Normativität der Sprache` eingebunden und wirklichkeitskonstituierend (Butler 2006: 211). Davon ausgehend, dass sich jeder Realitätsbezug aufgrund seiner Zitatförmigkeit auch auf diskursive Normen beziehen muss, d.h. immer in ein Feld bereits bestehender Symboliken und Semantiken eintritt, lassen sich Empörungswellen auch in Bezug auf die Absteckung geschlechtsspezifischer Normen hin untersuchen. Das sanktionierende und normierende Potenzial von mediatisierten Missachtungen entfaltet sich in der verletzenden Rede im weitesten Sinne, indem sie auch auf hegemoniale Geschlechterstereotype rekurieren und Subjekte »auf ihren Platz verweisen« (Butler 2006: 13). Die diffamierende Anrede mediatisierter Missachtung erkennt das adressierte Subjekt nicht an, sondern verwirft es und stellt somit potenziell dessen eigenes Sein in Frage. Wenn mediatisierte Empörungswellen wirklichkeitskonstituierend sind, gerät ihre Effektivität in den Fokus der Betrachtung. Die Effektivität mediatisierter Empörung lässt sich jedoch nicht vorhersehen. Insofern beabsichtigt die vorliegende Arbeit, perspektivische Streuungen zu generieren, also unterschiedliche Perspektiven miteinander zu diskutieren, welche die Prozesshaftigkeit mediatisierter Empörung betonen und ihren Ausgang prinzipiell offenlassen. Diese Perspektivierung betont folglich zugleich die Möglichkeit des Verfehlens ebendieser. Nicht jede Diffamierung funktioniert auch tatsächlich (vgl. Butler 2006: 32). Eine Arbeit über mediatisierte Missachtung hat sich somit der Aufgabe zu 
stellen, die Performativität mediatisierter Empörung im Kontext ihrer technologischen Bedingtheiten sowie ihrer Unkontrollierbarkeit zu diskutieren. Daran schließt sich unweigerlich die Frage an, inwiefern mediatisierter Missachtung Verletzungsmächtigkeit zugeschrieben werden kann. Es gilt zu fokussieren, welche Relationen und Gefüge aus dem Ineinandergreifen von Subjekten und Medientechnologien entstehen und in welcher Hinsicht mediatisierte Empörung ein Subjektivationsmodus darstellt, der im Spannungsfeld von Realität und Virtualität Wirksamkeiten entfaltet bzw. entfalten kann. Denn das Internet ist kein fiktiver Raum ohne Bedeutung für unsere Realität. Internetpraxis ist vielmehr konstitutiv für unsere Existenz. Wenn also Judith Butler konstatiert, »dass ich zu Beginn mein Verhältnis zu dir bin [Herv. i. Orig.], zweideutig angeredet und anredend, einem >Du< ausgeliefert, ohne das ich nicht sein kann und von dem mein Überleben abhängt« (Butler 2014a: 110), dann bedeutet dies, dass medientechnologisch bedingten Anredeszenarien, innerhalb derer Subjektivitäten erst ausgehandelt werden, existenziell bedeutsam sind. Zugleich bedeutet es den Abschied vom Konzept souveräner Subjektivität.

Die vorliegende Arbeit beabsichtigt entsprechend, einen Beitrag zur Verletzbarkeit im Kontext digitaler Medientechnologien zu leisten. Wenn Subjekte und ihre Körper nicht an ihrer Haut enden, sondern vielmehr ein Phänomen darstellen, welches immer auch erst durch performativ wirksame Intraaktionen ${ }^{6}$ von Mensch und Technologie im Entstehen begriffen ist, so drängt sich die Frage auf, wie eine potenziell gewaltsame Internetpraxis diese Medienkörper verändern, angreifen, verletzen kann. Damit positioniert sich die Konzeption verletzbarer Cyborgs, als die wir uns demnach allesamt verstehen können, gegen eine Position, die den realen, physischen Körper einem irrealen, virtuellen Körper gegenüberstellt. Die Folge ist eine theoretische Neujustierung, die von einem Körperverständnis ausgeht, welches über den >reinen` physischen Körper hinausgeht und stattdessen einem weiteren Verständnis von Materie im Spannungs-

6 Der Begriff Intraaktion ist von Karen Barad entlehnt und verweist auf die wechselseitige und aktive hervorgebrachte Verschränktheit mehrerer diskursiv-materieller Praktiken wie Apparate (Barad 2012a: 20f.). Damit betont der Begriff insbesondere die Praxisebene (vgl. Völker 2012: 22f.). Der physikalische Begriff Interferenz bringt jene Relationalität, verstanden als Verschränktheit und Überlagerung, ebenso in Anschlag, fokussiert jedoch eher auf das Spektrum, welches sich hieraus ergibt, weswegen insbesondere von Interferenzmustern geschrieben wird (vgl. Völker 2012: 24f.). Da es sich bei diesem Spektrum jedoch ebenso um eine relational und prozesshaft gedachte Verschränktheit handelt, sind beide Begriff nicht klar voneinander abgrenzbar. Vgl. vertiefend Kap. 2. 
feld von Realität und Virtualität folgt. Damit geht die Analyse mediatisierter Missachtung über Sprache und Schrift hinaus und diskutiert im Kontext ihrer technologischen Bedingtheit unausweichlich die Multimodalität des Phänomens, welche Anrufung somit unter hypermedialen Bedingungen hinterfragen muss (Eickelmann 2014b).

Vor diesem Hintergrund lässt sich die für die vorliegende Arbeit konstitutive Frage aufwerfen, wie mediatisierte Empörungswellen im Hinblick auf ihre geschlechterkonstituierende und -normierende Funktion einzuordnen sind; und damit untrennbar verbunden - wie gleichzeitig die Medialität des Internets konstituiert wird. Welche generative Rolle kommt dem Medialen in Bezug auf die Wirkmacht mediatisierter Empörung bzw. Missachtung zu, bedenkt man, »daß Worte, die eine Verletzung herbeiführen können, genauso gut ihr Ziel verfehlen und eine Wirkung herbeiführen können, die der beabsichtigten völlig zuwiderläuft«(Butler 2006: 139)? Aus diesen Fragen ergeben sich weiterführende Überlegungen, die in eine Konzeptualisierung von Verletzbarkeit im Spannungsfeld von Realität und Virtualität münden.

\section{Verlauf der Arbeit}

Kapitel 2 entwickelt zunächst die dieser Arbeit zugrunde liegende theoretische wie methodologische Perspektive. Ausgehend von der Frage nach der Verletzungsmacht mediatisierter Missachtung schlagen die hier entwickelten Überlegungen eine Konzeptualisierung von Verletzungsmacht im Spannungsfeld von Realität und Virtualität vor, die ihre ontologische Trennbarkeit negiert. Die Diskussion des Verhältnisses der Sphären >Realität` und >Virtualität $<$ bzw. >Materialität « und 〉Textualität` ist insofern für die vorliegende Arbeit zentral, als sie unweigerlich die Frage aufwirft, unter welchen Umständen eine wie auch immer geartete Äußerung materielle Effekte haben kann. Insbesondere im Kontext des New Materialism wird diese Frage behandelt, da sich ebendieser insbesondere der Aufgabe verschrieben hat, die angebliche Begrenztheit diskurstheoretischer Ausführungen zu überwinden und stattdessen zu betonen, dass die Welt nicht lediglich von sprachlichen bzw. textuellen Aushandlungsprozessen her gedacht werden kann, sondern dass vielmehr die Materialitäten, d.h. die Körper und Dinge im weitesten Sinne selbst als Effekt wie Agentien der prozesshaften Weltwerdung betrachtet werden müssen (vgl. Latour 1995/2016; Barad 1998/2007; Gehring 2007; Davis 2009; Busse 2013). Diese Überlegungen sollen mit dem semiotischen Ansatz Judith Butlers in einen produktiven Diskurs gebracht werden. Dieses >reading-of-insights-through-one-another< (Barad 2007: 71) soll zur Klärung der Frage dienlich sein, inwiefern mithilfe einer kritischen Relektüre des Agentiellen Realismus von Karen Barad und in Abgrenzung zur 
Symmetrischen Anthropologie von Bruno Latour eine Anreicherung der Arbeiten Judith Butlers erreicht werden kann, welche eben diese für die Perspektivierung und Analyse von Verletzungen im Spannungsfeld von Realität und Virtualität nutzbar macht. Unter Zuhilfenahme der Ausführungen von Elena Esposito, die eine Analyse und Konzeptualisierung unterschiedlicher Realitätsdimensionen vorgelegt hat, wird es möglich, theoretische und methodische Synergieeffekte herauszuarbeiten, die in einer Konzeption des Internets und der Partizipierenden als hybride Praxis münden, welche sich fortwährend entwickelt und in ihrem Prozessieren sowohl spezifische Konfigurationen von Subjektivität als auch von Medialität hervorbringt.

Methodologisch folgt aus diesen Ausführungen ein diffraktives Vorgehen, welches es erlaubt, die Relationalitäten unterschiedlicher Analyseebenen und Realitätsdimensionen miteinander zu diskutieren, ohne das zugrunde liegende Material erneut festzuschreiben. Im Gegensatz zur Reflexion versucht die Figur der Diffraktion eben nicht, ein Abbild eines vermeintlich seienden Phänomens herzustellen, sondern distanziert sich notwendigerweise von ihrem Gegenstand (Deuber-Mankowsky 2011b: 89f.). Mediatisierte Missachtung mithilfe einer diffraktiven Methode zu beschreiben erlaubt es, das Ineinandergreifen unterschiedlicher Ebenen, wie beispielsweise Gender und Medium, das Menschliche und das Technische, als spezifisches relationales Gefüge darzustellen und so einen Beitrag dazu zu leisten, spezifische Intraaktionen bzw. Interferenzen nicht etwa in der Analyse wieder zu trennen, sondern konsequent als sich wechselseitig performativ herstellende Aspekte, die nicht unabhängig voneinander diskutiert werden können, in ihrem gegenseitigen Werden zu betrachten. Eine diffraktive Methodologie entwirft selbst Interferenzmuster, ohne sich einem spezifischen Wahrheitsregime zu verpflichten (vgl. Trinkaus 2013: 154). Damit strebt diese theoretische und methodologische Verortung an, einen Beitrag für eine Begründungslogik zu leisten, die jenseits der Idee einer metaphysischen Weltordnung arbeitet. Das vorläufige Ziel dieses Kapitels ist eine Konzeptualisierung verletzbarer Cyborgs, welche die genealogische Verbindung von Subjektivität und Technizität zum Ausgangspunkt nimmt, um Verletzbarkeiten in diesem Kontext auf der einen Seite beschreib- und diskutierbar zu machen. Auf der anderen Seite geht es zudem darum, die Kontingenz und Instabilität eben jener Prozesse zu betonen, so dass hieraus eine andere Perspektive auf Handlungsfähigkeit und Widerstand erwächst.

Diese Erörterungen zur Grundlage nehmend, fokussiert Kapitel 3 Prozesse der Subjektivation im Netz. In einem ersten Schritt wird hier, aufbauend auf einer diskursiv-materiellen Geschichte des Internets, danach gefragt, welche zentralen Aspekte die Kultur der Dauerkonnektivität (van Dijck 2013) auszeich- 
nen und wie jene Aspekte in Relation zueinander wirken. Die Herausarbeitung der Aspekte digitaler Hypermedialität, Daueradressierbarkeit, Beschleunigung und Distanzabbau verbindet diskursive wie materielle Aspekte, indem diskursive Rahmungen, technische Bedingungen sowie nutzungspraktische Effekte zusammengedacht werden. In einem zweiten Schritt wird näher auf die unter jenen Bedingungen entstehenden Subjektivitäten fokussiert, die sich im Spannungsfeld von Mensch und Technologie entfalten und als produktive Machteffekte gelesen werden können. Die Trennung von Mensch und Technologie, u.a. dargestellt am Beispiel der Differenz von Mensch und Social Bot, rückt einmal mehr als Effekt agentieller Schnitte in den Blick, die nicht zuletzt die Intraaktion von Mensch und Medientechnologie ontologisch trennen. In einem dritten Schritt werden die Ausführungen für die Perspektivierung mediatisierter Empörungswellen als Subjektivationsmodus fruchtbar gemacht, woraus eine Perspektive auf Verletzbarkeit in der Kultur der Dauerkonnektivität abgeleitet wird.

In Anlehnung an die vorangegangenen Ausführungen werden in Kapitel 4 jene Diskursstränge einem diffraktiven Leseprozess unterworfen, welche das Verhältnis des Medialen und seiner potenziellen Gewaltförmigkeit thematisieren. Hierbei geht es unausweichlich um die Frage, inwiefern diffamierende Adressierungen im Netz als $\gg$ Hassrede aufgefasst werden oder als Ausdruck der $>$ Redefreiheit<. Denn die Einschätzung der Verletzungsmacht mediatisierter Missachtung, welche in spezifischen Diskursen unterstellt bzw. negiert wird, hat wiederum diskursiv-materielle Effekte, sowohl im juristischen als auch politischen Sinne. Auf der Grundlage der Diskussion der vermeintlichen Opposition von Diskursen, die mediatisierte Missachtung als `Hassrede` verorten und jenen, die mediatisierte Missachtung als Aspekt von >Redefreiheit`verstehen, wird gezeigt werden, dass beide Seiten der Medaille an einem konsensualen Universalismus orientiert sind, der tendenziell die Juridifizierung mediatisierter Missachtung sowie eine Entpolitisierung des Internets stützt. Entsprechend wird argumentiert, dass jene Opposition, welche die Diskurse rund um das Thema mediatisierte Missachtung beherrscht und nichts außerhalb von starren Gewalt- und Freiheitsentwürfen kennt, einen Aspekt der Herstellung souveräner Subjektivitäten darstellt. Entweder, weil die Adressierenden per se als souverän verstanden werden und folglich unmittelbar in der diffamierenden Adressierung einen Gewaltakt vollziehen (〉Hassrede`), oder eben, weil die Adressierenden als souverän diskursiviert werden, die im Sinne eines Freiheitsideals grenzenlose Expressivität einfordern, im Sinne von: >Das wird man ja wohl mal sagen dürfen ২ (>Redefreiheit ). Mit Rekurs auf die Souveränitätskritik von Judith Butler soll eine alternative Sichtweise auf die Effektivität mediatisierter Missachtung entwickelt werden, welche die Opposition von >Hassrede` und >Redefreiheit` überwindet 
und diese damit weder per se als Gewaltakt deklariert, noch ihre machtvollen und potenziell verletzenden Effekte verneint.

Kapitel 5 greift die oben bereits angesprochenen Figurationen mediatisierter Empörung auf, die in der diskursiven Verhandlung von mediatisierter Missachtung im Netz relevant gemacht werden. Geleitet von der Frage, wie die Figurationen >Trollen`, >Cybermobbing « und `Shitstorm` diskursiv unterschieden werden, aber auch, inwiefern ihnen ähnliche Stoßrichtungen zugrunde liegen, beabsichtigt das Kapitel, eine Diskussion der unterschiedlichen figurativen Konstellationen mediatisierter Missachtung zu leisten. Diese sowohl diskursanalytisch als auch an der Methodologie der Diffraktion orientierten Erörterungen dienen als Grundlage, um bei den folgenden Fallanalysen eben jene diskursiven Rahmungen kritisch daraufhin betrachten zu können, wie sie wirklichkeitskonstituierend bei spezifischen Fällen von mediatisierter Missachtung wirksam werden. So macht es einen enormen Unterschied, ob ein spezifischer Sachverhalt bzw. eine spezifische Narration im Kontext von 〉Shitstorms`, 〉Cybermobbing ২ oder auch >Trollen` verhandelt wird. Während der Begriff >Shitstorm` tendenziell zwar die Medienspezifizität mediatisierter Missachtung anspricht, so trägt er im gleichen Zuge dazu bei, Gatekeeper_innen und Kommunikationsberater_innen zu mobilisieren, die diesem Phänomen Einhalt gebieten sollen. Der Begriff >Cybermobbing ist wiederum anders gelagert, da er insbesondere im Rahmen eines pädagogisierten und psychologisierten Diskurses den >Opferschutzく fokussiert. Der Begriff des >Trollens meintliche politische Potenzial eben jener mediatisierten Missachtung und trägt so zu einer Trivialisierung bzw. Politisierung des Phänomens bei. Es lässt sich also zeigen, dass die Begriffe, die in den Diskursen über mediatisierte Missachtung verhandelt werden, jeweils unterschiedliche Phänomene zur Folge haben. Je nachdem also, wie mediatisierte Empörungswellen verhandelt werden, stellt sich die Frage nach der Verletzungsmacht und ihrer Bedeutung für Subjektivationsprozesse anders.

Kapitel 6 lässt sich als vertiefende Erprobung der zuvor entwickelten Konzeption mediatisierter Missachtung samt der mit ihr einhergehenden diskursiven Rahmungen wie Materialitäten verstehen. In einem ersten Schritt wird das Design diffraktiver Fallanalysen vorgestellt, welches die in den Fokus geratenen Fälle als Phänomene fasst. Mithilfe der diffraktiven Analyse des zugrunde gelegten Materials wird die Verletzungsmacht mediatisierter Missachtung als Effekt komplexer Relationalitäten herausgearbeitet. Bei den beiden diffraktiven Fallanalysen handelt es sich um jeweils eine Geschichte zweier Phänomene mediatisierter Missachtung, die sich insbesondere durch voneinander unterscheidbare Relationalitäten auszeichnen. Die erste Fallanalyse bezieht sich auf den Fall 
Anita Sarkeesian, die aufgrund ihrer Webserie >Tropes vs. Women in Video Games`, in der sie auf Geschlechterstereotype in digitalen Spielen aufmerksam macht, mediatisierter Missachtung ausgesetzt ist. Im Kontext der Debatten um \#Gamergate entzündet sich die Debatte um Feminismus und digitale Spiele erneut. Der Fall eignet sich für Analyse für die hier vorliegende Fragestellung insbesondere deswegen, da er zum einen die konstitutive Dimension der Kategorie Geschlecht verdeutlicht, als auch die Multimodalität mediatisierter Missachtung offenlegt. Die zweite Fallanalyse bezieht sich auf den Fall Amanda Todd. Hier geht es um die Diskussion der Geschichte einer 15-Jährigen, die nach einer jahrelang andauernden Leidensgeschichte eben diese auf YOUTUBE sichtbar machte. Was folgte, war nicht etwa ausschließlich Mitgefühl, sondern multimodale mediatisierte Missachtung. Diese Geschichte zeigt auf gnadenlose Weise, inwiefern erstens die Betrauerbarkeit von Subjektivitäten im Netz zugunsten neoliberaler Diskurse verneint wird und zweitens, dass mediatisierte Missachtung jederzeit in mediatisierte Gewalt umkippen kann, nämlich genau dann, wenn Geschlechternormen keine andere Möglichkeit mehr einräumen, als den vermeintlich selbst gewählten Tod. In einer zuspitzenden Zusammenfassung werden beide Fallanalysen übereinandergelegt. Es erfolgt ein Resümee darüber, inwiefern die Feinanalyse der beiden Phänome Erkenntnisse zur der Frage liefern, welche Verletzbarkeit mediatisierte Missachtung im Netz adressiert.

Bernhard Pörksen und Hanne Detel haben auf die Gefahr hingewiesen, dass die wissenschaftliche Auseinandersetzung mit Verletzbarkeiten eben jene zu diskutierenden Verletzungen wiederholen kann:

»Es wird nämlich deutlich, dass die Analyse einer angeblichen Normverletzung unvermeidlich diese Normen reproduziert - und die Gefahr erzeugt, die Schmähung, nur eben unter dem Deckmantel von Information, Aufklärung und Analyse, ein weiteres Mal zu wiederholen« (Pörksen/Detel 2012: 17).

Dieser wichtige Hinweis soll hier nicht ungeachtet bleiben, allerdings möchte ich mit Judith Butler davon ausgehen, dass Wiederholen immer ein Verschieben bedeutet, so dass die Anführung jener hier zu diskutierenden Beispiele und (Lebens-)Geschichten eben nicht die Schmähungen zu reproduzieren sucht. Denn auch wenn die hier zur Disposition stehenden Phänomene ein Trauma in sich tragen - so scheint die verrückende Wiederholung ein vielversprechendes Instrument für die wissenschaftliche Auseinandersetzung mit eben jenen unsagbaren Phänomenen zu sein (vgl. Butler 2006: 66). 